\section{P.2.14 PULMONARY DYSFUNCTION IN INDIUM TIN OXIDE EXPOSED WORKERS}

${ }^{1}$ Saou-hsing Liou*, ${ }^{2}$ Yuan-Ting Hsu, ${ }^{1}$ Wei-Jin Li, ${ }^{1}$ Wei-Te Wu. ${ }^{1}$ National Health Research Institutes, Miaoli County, Taiwan; ${ }^{2}$ Graduate Institute of Life Sciences, National Defense Medical Center, Taipei, Taiwan, ROC

\subsection{6/OEM-2019-EPI.247}

Aim To investigate the relationship between indium exposure and lung effects markers among indium tin oxide (ITO) manufacturing workers without job change.

Methods We enrolled 179 male workers from ITO target manufacturing and recycling factories in Taiwan. Plasma indium (P-In), urine indium (U-In) and creatinine adjusted UIn (U-In/Creat.) were used as internal dose of indium exposure. Plasma Krebs von den Lungen-6 (KL-6) and surfactant protein D (SP-D) were used as markers of interstitial pneumonitis. Forced vital capacity (FVC), forced expiratory volume at 1 st second (FEV1), and FEV1/FVC were also evaluated by spirometry.

Results After adjusted for covariates by linear regression, plasma, urinary and creatinine adjusted indium were increased in high exposure group (P-In: $\beta=1.13, \quad \mathrm{p}<0.001$; U-In: $\beta=0.54, p<0.05$; U-In/Cre: $\beta=0.63, p<0.01$ ) and low exposure group (P-In: $\beta=0.75, \quad p<0.05 ; \quad U-I n / C r e: \quad \beta=0.52$, $\mathrm{p}<0.05)$ with comparison to reference group. Plasma KL-6 was higher in high exposure group $(\beta=0.24, \mathrm{p}<0.05)$ compared to reference group, but not for surfactant protein D (SP-D). Furthermore, FVC and FEV1 were reduced in both high exposure group (FVC: $\beta=-0.08, \quad \mathrm{p}<0.01$; FEV1: $\beta=-0.05, p<0.05$ ) and low exposure group (FVC: $\beta=-0.06$, $\mathrm{p}<0.05)$ compared to reference group.

Conclusion Our findings indicate indium exposure was related to restrictive lung dysfunction, decreased lung function for both FEV1 and FVC test but not for FEV1/FVC ratio. Meanwhile, increased plasma KL-6 in high exposure group also supports that indium exposure results in increased risk of interstitial pneumonitis among direct indium exposure workers. Our study provided an explanation to the consequence of indium exposure- interstitial pneumonitis-restrictive lung dysfunction.

\section{P.2.15 EXPOSURE TO 2,3,7,8-TETRACHLORODIBENZO-P-DIOXIN AND MORTALITY AT A TRICHLOROPHENOL PLANT IN NEW ZEALAND}

'David Mcbride*, ${ }^{2}$ James Collins, ${ }^{3}$ Thomas Bender, ${ }^{3}$ Kenneth Bodner, ${ }^{4}$ Lesa Aylward. ${ }^{1}$ University of Otago, Dunedin, New Zealand; ${ }^{2}$ Saginaw Valley State University, Saginaw, USA; ${ }^{3}$ Dow Chemical, Midland, USA; ${ }^{4}$ Summit Toxicology, Falls Church, USA

\subsection{6/OEM-2019-EPI.248}

Objectives To describe how 2,3,7,8-tetrachlorodibenzo-p-dioxin (TCDD) exposure influenced mortality in a cohort of workers exposed more recently, and at lower levels, than other cohorts of trichlorophenol process workers.

Methods A cohort study of 1599 men and women working between January 1, 1969 and November 1, 1988 at a plant producing the herbicide 2,4,5-trichlorophenoxyacetic acid $(2,4,5 \mathrm{~T})$ with TCDD as a contaminant.

A toxicokinetic model developed in a previous follow up was updated to estimate cumulative TCDD exposure for each individual in the study. Calculation of cause-specific standardized mortality ratios (SMRs) and 95\% confidence intervals (95\%CIs) compared those never and ever exposed to TCDD. Dose-response trends were assessed firstly through SMRs stratified in quartiles of cumulative TCCD exposure, and secondly with a proportional hazards model.

Results The toxicokinetic model intercept of 5.1 parts per trillion (ppt) of TCDD was consistent with background New Zealand TCDD concentrations among older members of the population. Exposed workers had non-significant increases in all cancer deaths $\mathrm{SMR}=1.08$, 95\% CI 0.86-1.34, deaths from soft tissue sarcoma, $\mathrm{SMR}=2.38,95 \% \mathrm{CI}$ : $0.06-13.26$, nonHodgkin lymphoma, SMR =1.57, 95\% CI: 0.32-4.59, diabetes, $\mathrm{SMR}=1.27,95 \% \mathrm{CI}: 0.55-2.50$ and ischaemic heart disease, $\mathrm{SMR}=1.21, \quad 95 \% \mathrm{CI}:$ 0.96-1.50. Lung cancer deaths $\mathrm{SMR}=0.95$, 95\% CI: 0.56-1.53, were fewer than expected. Neither the stratified SMR nor proportional hazard analysis showed a dose response relationship.

Conclusion We found neither an excess of all cancers, or any specific cancer, nor a trend with TCDD exposure. This argues against the carcinogenicity of TCDD at lower levels of exposure.

\section{P.2.16 HEALTH AND PRODUCTIVITY: A FIVE YEAR STUDY (2010-14) IN A LARGE AUTOMOBILE INDUSTRY IN INDIA}

${ }^{1}$ Gautham Sukumar*, ${ }^{2}$ Kowshik Kupatira, ${ }^{1}$ Pradeep Banandur, ${ }^{1} \mathrm{G}$ Gururaj. ${ }^{1}$ National Institute of Mental Health and Neuro Sciences, Bangalore, India; ${ }^{2}$ Leading automobile industry, Bangalore, India

\subsection{6/OEM-2019-EPI.249}

Introduction This 'Health and productivity' project was implemented to identify priority health problems and health related productivity loss in a large automobile industry in India. It hopes to re-emphasize that 'OH department' is a partner in productivity.

Objectives

1. To estimate prevalence, incidence rates, trends and risk for health problems among employees (year 2010-14)

2. To identify leading causes of hospitalization and out-patient care among employees (year 2010-14).

3. To quantify loss in productive work time attributed to health related absence in year 2014 and forecast to year 2025

Methods A five year records analysis (2010-14) was conducted in a leading automobile industry in year 2015-16. Data was pooled from clinic visit records, annual medical examination , insurance claims and leave records, systematically using employee ID. Trends and incidence rates of leading health risks were computed per 1000 person-months of employment. Leading causes and trends in out-patient and in-patient care of employees were determined. Total work time (man-hours) was computed and health related loss in work-time was derived. Cox-regression was used to assess risk between work department and morbidity. ARIMA method was used to forecast productivity loss till year 2025 .

Results Between 2010-14, overweight and hypertension were identified as leading health risks. Respiratory, musculoskeletal and digestive disorders were leading causes for clinic visits. Infectious diseases are leading cause for hospitalization. Health related absence accounted for $1.87 \%$ of total productive time. Forecasting indicates an increase to $9.31 \%$ by year 2025 . 
Conclusion Health related absence contributes to significant loss in productive work-time. There is scope to reduce these losses by improvement in health situation. Our study provided key information and trends for planning evidence based health promotion programmes in the industry.

\section{P.2.17 CASE REPORT: THREE OCCUPATIONAL DISEASES IN A NAIL TECHNICIAN} 1,2Shlomo Moshe*, 1,2 Ayala Krakov. 'Maccabi Healthcare Services, Holon, Israel; ${ }^{2}$ Sackler
Faculty of Medicine, School of Public Health, Tel-Aviv University, Tel Aviv, Israel

\subsection{6/OEM-2019-EPI.250}

Introduction In cosmetics, acrylic glues are used to apply nail and eyelash extensions. Acrylates are highly irritant materials and may cause local irritation (Contact Dermatitis - CD), respiratory irritation (Asthma) and systemic irritation (Urticaria). This case report describes these three occupational diseases.

Clinical presentation A 48-year-old female, normally healthy, has been working in the nail beauty field for 11 years. Lately, following an increase of work, she started coughing every morning and feeling shortness of breath which was worsen at the start of work, and improved on vacations. In addition, the patient suffered from skin tenderness in her fingers. An examination of the hands showed a typical picture of CD in left hand fingers 4-5.

Methods and results Specific Patch Tests showed sensitivity to Hydroxyethyl Methacrylate and Hydroxypropyl Methacrylate. A methacholine challenge test showed a $22 \%$ decrease in FEV1 at $3.94 \mu \mathrm{mol}$ methacholine (i.e. positive test). The patient performed a Peak Expiratory Flow test (PEF). After a week in the nail salon her respiratory and dermatological status worsened greatly, including development of contact urticaria. She was instructed to stop immediately the exposure and start taking $\mathrm{p} / \mathrm{o}$ steroids. Due to her 3 occupational diseases she was removed from work and latter was recognized as having 3 occupational disease by the Israeli National Insurance which supported her vocational rehabilitation.

Discussion Acrylic glues are hazardous agents which can cause several occupational diseases and require occupational environment monitoring yearly according to Israeli regulations. The TLV-TWA is 2ppm. Small private businesses like the patients' place don't perform environmental monitoring or use personal or environmental protective equipment which endanger its workers (usually low social class women) who are not aware of the dangers in this industry. More public attention should be given to the risk in this industry.

\section{P.2.18 THE CURRICULUM IMPLEMENTATION OF OCCUPATIONAL MEDICINE IN ISRAEL}

Shlomo Moshe*. 'Maccabi Healthcare Services, Occupational Medicine Department, Holon, Israel; ${ }^{2}$ Sackler Faculty of Medicine, School of Public Health, Department of Environmental and Occupational Health, Tel Aviv University, Tel Aviv, Israel

\subsection{6/OEM-2019-EPI.251}

Background At the early 1990s occupational medicine (OM) was recognized as a specialty in Israel. Fifteen years later after gathering problems and shortcomings of the first curriculum, the Israeli Association of occupational medicine (IAOM) defined the framework for a new curriculum in order to have a modern and better curriculum.
Methods In the early 2000 an expert committee apointed by the IAOM suggested a new sylabous, based on the scientific literature. The recommendations were sent to all experts in $\mathrm{OM}$ in Israel, and other proffesional members like industrial hygienist. We got several responses. Most people suggested additional standards.

Results The curiculun includes 54 months of training programme. The characteristics of the new curriculum are: interaction between theory and practice; fields of knowledge, learning process; competencies which are needed; and multidisciplinarity. We added an obligatory walkthroug list of industries. Three of the walkthroug reports consists a part of the final oral examinations.

Conclusions The aim of the curriculum was to produce a comprhensive aproach in OM competencies. The curiculum was adopted by the Israeli Medical Association and Ministery of Health since 2010.

\section{P.2.19 EFFECT OF COLLABORATIVE SUPPORT OF OCCUPATIONAL AND CLINICAL PHYSICIANS - EVALUATION OF OUTCOME DUE TO TREATMENT DROP OUT IN NON-COMMUNICABLE DISEASES}

${ }^{1,2}$ Go Muto. 'Harvard T.H.Chan School of Public Health, Boston, USA; ${ }^{2}$ Kitasato University, Dep of Hygiene, School of Medicine, Sagamihara/Kanagawa, Japan

\subsection{6/OEM-2019-EPI.252}

Objectives To develop a prediction model of outcome due to drop out in treatment for non-communicable diseases (NCDs) in Japan as well as to evaluate the effectiveness of collaboration between occupational and clinical physicians.

Methods Using both claim and health check-up data (JMDC) of beneficiaries aged 20 to 74 years who were covered by health insurance societies between 2008 and 2016, we identified high risk 533955 examinees of health checkups who met the following criteria and had no claim data on the corresponding items 4 months prior to the health checkups. The criteria was either blood pressure, blood glucose level, or blood lipid level provided by Japanese government.

Results The cumulative non-visiting rates at 12 months after health check-ups were $84.4 \%$. The rate of drop off during treatment was over 70\%. As for HT, DM, and DL, the cumulative non-visiting rates at 12 months were 84.3, 67.9, and $86.1 \%$, respectively. When focusing on the high risk group, the rate was $74.0 \%$, since the rate for extremely high risk DM $(\mathrm{HbA} 1 \mathrm{c} \geq 8.4 \%)$ was $51.9 \%$.

Conclusion Over $80 \%$ high risk examinees of non-communicable diseases did not visit to medical institutions during 1 year period after health check-ups, although approximately half of the individuals with extremely high risk of DM visited to physicians at least one time. Finally, we developed a predictive scoring model for visiting to medical institutions as well as the risk of drop out from treatment, and it was well validated from both discrimination and calibration viewpoints. In addition, we evaluated the effectiveness of strategy for preventing treatment drop off through collaborative support from occupational and clinical physicians. This strategy enables public/occupational health staffs to individualize population for strong intervention and to support harmonization of treatment and work in NCDs. 